\title{
Effects of puerarin on spatial learning and memory function in mice with acute alcohol consumption: An evaluation based upon firing rate and oxygen saturation analysis
}

\author{
Weitao Li ${ }^{A, D, F}$, Yan Zhang ${ }^{B}$, Yanbai Xue ${ }^{B, C}$, Hejuan Yu ${ }^{B, C}$, Yameng Zhang ${ }^{B}$, Ling Tao ${ }^{E}$, Yamin Yang ${ }^{D, E}$, Zhiyu Qian ${ }^{A, F}$ \\ Department of Biomedical Engineering, College of Automation Engineering, Nanjing University of Aeronautics and Astronautics, China \\ A - research concept and design; B - collection and/or assembly of data; C - data analysis and interpretation; \\ $D$ - writing the article; $E$ - critical revision of the article; $F$ - final approval of the article
}

Address for correspondence

Weitao Li

E-mail: liweitao@nuaa.edu.cn

\section{Funding sources}

National Natural Science Foundation of China (81727804 and 81601532), Jiangsu Science and Technology Support Plan (Social Development) (BE2016759), Natural Science Foundation of Jiangsu Province (BK20160814), and Scientific Research Foundation of Nanjing University of Aeronautics and Astronautics (YAH16009).

Conflict of interest

None declared

Received on April 27, 2017

Reviewed on August 18, 2017

Accepted on December 21, 2017

Published online on October 9, 2018

\section{Cite as}

Li W, Zhang Y, Xue Y, et al. Effects of puerarin on spatial learning and memory function in mice with acute alcohol consumption: An evaluation based upon firing rate and oxygen saturation analysis. Adv Clin Exp Med. 2019;28(2):171-178. doi:10.17219/acem/81520

DOI

10.17219/acem/81520

\section{Copyright}

Copyright by Author(s)

This is an article distributed under the terms of the

Creative Commons Attribution Non-Commercial License

(http://creativecommons.org/licenses/by-nc-nd/4.0/)

\begin{abstract}
Background. Ethanol is associated with various medical comorbidities affecting the brain and central nervous system.

Objectives. The use of puerarin in treating alcohol-induced memory disorders is systematically evaluated in this study based upon an analysis of the firing rate and oxygen saturation $\left(\mathrm{SO}_{2}\right)$.

Material and methods. A multi-channel data acquisition system and near-infrared spectroscopy (NIRS) were combined to obtain the electroneurophysiological signals and $\mathrm{SO}_{2}$ in the hippocampus of mice from the Institute of Cancer Research (ICR) after ethanol injection. A T-maze test was performed to study the alteration of spatial memory function. Ethanol was administered intraperitoneally (i.p.) in 2 dosages $(1.5 \mathrm{~g} / \mathrm{kg}$ and $0.5 \mathrm{~g} / \mathrm{kg})$. To investigate the effects of puerarin against acute ethanol-induced memory impairment, the same parameters corresponding to electroneurophysiological signals, $\mathrm{SO}_{2}$ and behavior performance in mice were also recorded upon the supplementation of puerarin in 2 dosages (25 mg/kg and $50 \mathrm{mg} / \mathrm{kg}$ ).

Results. An inhibited firing rate and decreased $\mathrm{SO}_{2}$ were found in mice treated with an ethanol dose of $1.5 \mathrm{~g} / \mathrm{kg}$, which resulted in a low correct choice rate in the T-maze test, while an opposite trend appeared in mice which had been administered a moderate ethanol dose $(0.5 \mathrm{~g} / \mathrm{kg})$. The trend of $\mathrm{SO}_{2}$ was positively correlated with that of the firing rate. A decreased firing rate and $\mathrm{SO}_{2}$ were accompanied by a decrease in the correct choice rate. With the supplementation of puerarin, a significant increase in the mean firing rate and $\mathrm{SO}_{2}$, as well as an improved correct choice rate, can be found in mice injected with excessive ethanol.

Conclusions. The electroneurophysiological signals and NIRS were combined for the first time to prove that an excessive intake of ethanol can inhibit the spatial learning and memory function of mice. The supplementation of puerarin can suppress these adverse effects induced by ethanol at a high dosage, as evidenced by the increased firing rate and $\mathrm{SO}_{2}$.
\end{abstract}

Key words: spatial memory, near-infrared spectroscopy, hippocampal neuron, microelectrode 


\section{Introduction}

Alcohol abuse and alcoholism are serious public health problems throughout the world. Ethanol is associated with various medical comorbidities affecting numerous body systems, such as the brain and central nervous system. ${ }^{1}$ The hippocampus, which is closely related to learning and memory function, can especially be affected by ethanol. ${ }^{2,3}$ Thus, developing suitable medications and evaluating their use in the treatment of alcohol-induced disorders remains a challenging goal in alcohol-related research.

As a Chinese medicinal herb, Puerariae radix has been proven to reduce alcohol consumption in both humans and animals. ${ }^{4,5}$ Puerarin is one of the main effective components of Puerariae radix. ${ }^{6}$ It has been found that puerarin can inhibit oxidative stress induced by acute alcoholism. ${ }^{7}$ Several studies have reported that the supplementation of a Puerariae radix-ethanol extract can inhibit lipid peroxidation in the liver and enhance the antioxidative defense competence of rats. ${ }^{8}$ In addition, many studies have indicated that puerarin may ameliorate hippocampal neuronal death induced by oxygen/glucose deprivation in vitro and may improve the learning-memory ability after global cerebral ischemia and reperfusion in rats. ${ }^{4,9}$

Mounting evidence suggests that alcohol affects brain function by interacting with multiple neurotransmitter systems in the hippocampus. ${ }^{10}$ It has been shown that the effects of ethanol on brain function are related to altered hippocampal physiology. However, the way the neurotransmitter systems are affected following medication (e.g., puerarin) for acute alcoholism, and how these changes in neurotransmission contribute to the protective effects of such medication, are poorly understood.

Electrophysiological technology using a microelectrode array can provide an important basis for the study of brain neural activities. ${ }^{11,12}$ This technology could obtain highresolution temporal and spatial response patterns of information in neural networks. ${ }^{13}$ In addition, the distribution and change of blood oxygen in the cerebral cortex could be another indicator for understanding the activity of the brain under the influence of alcohol. ${ }^{14}$ By using near-infrared spectroscopy (NIRS) noninvasively, oxygen saturation $\left(\mathrm{SO}_{2}\right)$ can be measured simultaneously while acquiring hippocampal neurophysiological data.

In this study, in order to investigate the effects of puerarin on the memory system of mice following acute alcohol consumption, microelectrodes and NIRS were combined in order to obtain the neurophysiological signals and $\mathrm{SO}_{2}$ in the hippocampus, and a T-maze test was performed to evaluate the functioning of spatial memory. This research presents a novel approach to evaluating the protective effects of puerarin against acute ethanol-induced memory impairment in mice.

\section{Material and methods}

\section{Animal preparation}

The Institute of Cancer Research (ICR) male mice ( 32 g) were purchased from the Qinglongshan Animal Experiment Center (Nanjing, China). After individualization (postnatal day 36), mice were housed in cages at a constant temperature $\left(25 \pm 1^{\circ} \mathrm{C}\right)$ and controlled illumination (12-h light/dark cycle) and humidity (55 $\pm 10 \%)$ for 7 days. Food and water were available ad libitum. All animal experimental procedures were conducted in accordance with the guidelines of the protocols approved by the Institution Animal Care and Use Committee at Nanjing University of Aeronautics and Astronautics, China.

The 9-week-old mice were randomly assigned to 8 groups and each group consisted of 8 mice. According to previous studies, the effective doses of puerarin mostly ranged from 25 to $100 \mathrm{mg} / \mathrm{kg} .{ }^{8}$ Therefore, in this study, dosages of 25 and $50 \mathrm{mg} / \mathrm{kg}$ of puerarin were chosen and all medications were injected intraperitoneally (ip.). For clarification, the groups of our experimental design are listed in Table 1. As control groups, the mice in groups $\mathrm{G} 1$ and $\mathrm{G} 2$ were injected with saline $(1.5 \mathrm{~g} / \mathrm{kg})$ and puerarin $(50 \mathrm{mg} / \mathrm{kg})$, respectively. Groups G3-G5 and groups G6-G8 were defined as high-ethanol-dose groups and low-ethanol-dose groups, respectively. Groups G3 and G6 were injected with ethanol alone at different concentrations. Groups G4 and G5 were administered puerarin in doses of 25 and $50 \mathrm{mg} / \mathrm{kg}$, respectively, $20 \mathrm{~min}$ after the injection of ethanol $(1.5 \mathrm{~g} / \mathrm{kg})$. In groups $\mathrm{G} 7$ and $\mathrm{G} 8$, puerarin was administered in doses of 25 and $50 \mathrm{mg} / \mathrm{kg}$, respectively, after the injection of ethanol at a lower dose $(0.5 \mathrm{~g} / \mathrm{kg})$.

The mice were anesthetized by an initial injection i.p. of $5 \%$ chloral hydrate $(\sim 400 \mathrm{mg} / \mathrm{kg})$. Then, the mice were placed in a stereotaxic frame with a skull-reduced flat orientation. An incision was made in the skin and a bur hole was drilled over the right side of the hippocampus after cleaning the skull surface. The center of the hole was roughly $1.8 \mathrm{~mm}$ posterior to the bregma and $1.5 \mathrm{~mm}$ lateral to the midline. Afterwards, the mice were implanted with a $2 \times 4$ nickel 14-cadmium microelectrode array (diameter:

Table 1. Groups of experimental design

\begin{tabular}{|l|c|c|}
\multicolumn{1}{|c|}{ Groups } & Doses [g/kg b.w.] & $\begin{array}{c}\text { Number } \\
\text { of animals }\end{array}$ \\
\hline G1 & saline (1.5) & 8 \\
\hline G2 & puerarin (0.05) & 8 \\
\hline G3 & ethanol (1.5) & 8 \\
\hline G4 & ethanol (1.5) and puerarin (0.025) & 8 \\
\hline G5 & ethanol (1.5) and puerarin (0.05) & 8 \\
\hline G6 & ethanol (0.5) & 8 \\
\hline G7 & ethanol (0.5) and puerarin (0.025) & 8 \\
\hline G8 & ethanol (0.5) and puerarin (0.05) & 8 \\
\hline
\end{tabular}


$\sim 33 \mu \mathrm{m}$, impedance: <1 $\mathrm{M} \Omega$ ), targeting the hippocampal region (1-2 $\mathrm{mm}$ in depth). In addition, the NIRS probe was inserted in the adjacent region of the hippocampus.

\section{Electrophysiological signal recording}

After the mice awoke from anesthesia, 8-channel local field potentials (LFPs) and spikes were recorded simultaneously using a Cerebus Multi-Channel Acquisition System (Cyberkinetics, Foxborough, USA). The LFP data was amplified (gain: 5000), filtered $(0.3-500 \mathrm{~Hz})$ and sampled at $2 \mathrm{kHz}$. Spikes (high pass filter: $250-7500 \mathrm{~Hz}$, sampled at $30 \mathrm{kHz}$ ) with a root mean square exceeding 5.0 were stored with the time stamps per channel. As shown in Fig. 1A, the system consists of an amplifier power supply, a neural signal amplifier, a processor, and a computer system. The location of the 8-channel microelectrode in the hippocampus of the brain is illustrated in Fig. $1 C$. The procedure of ethanol and puerarin injection and measurement is shown in the upper row of Fig. 1D, and the dash circles indicate the location of the hole in the skull.

Prior to any intraperitoneal injection, a 5-min baseline of a spontaneous neural electrophysiological signal was first recorded. Neural activity recording started upon the injection of ethanol and continued for $80 \mathrm{~min}$. Four spontaneous electrophysiological signals at a bin width of $5 \mathrm{~min}$ were stored with a time interval of $20 \mathrm{~min}$.

\section{Spike recording and data analysis}

Spike acquisition and collection was carried out with a Cerebus system (Blackrock Microsystems LLC, Salt Lake City, USA). To obtain a spike signal, the wide-band electrophysiological signals were filtered at $0.25-5 \mathrm{kHz}$. The power (root mean square) of the filtered signal in a sliding window of $0.2 \mathrm{~ms}$ was calculated for spike detection. Spikes with a power of more than 5 times the standard deviation (SD) from the baseline mean were extracted. The spike waveforms were reconstructed to $30 \mathrm{kHz}$ based on the sampling theorem. The units were then identified and de-noised by clustering software Wave_Clus (University of Leicester, UK). The sorting neuron firing rate was obtained at a bin width of $10 \mathrm{~s}$.

\section{Oxygen saturation measurement}

Along with electrophysiological signal recording, $\mathrm{SO}_{2}$ in the hippocampus of the mice was simultaneously measured using a customized NIRS monitoring system developed by our lab. The schematic design of the NIRS system is shown in Fig. 1B. The system consists of an integrated double optical fiber probe (diameter: $200 \mathrm{um}$ ), a halogen light source (HL2000-HP-FHSA; Ocean Optics, Largo, USA), a fiber optic spectrometer (USB2000; Ocean Optics), and a computer with custom monitoring software (Laboratory of Biomedical Photonics, Nanjing University of Aeronautics and Astronautics, China). ${ }^{15,16} \mathrm{The}_{2}$ value of the NIRS monitoring system was validated by an ISS Oximeter (96208; ISS, Champaign, USA). ${ }^{17}$ Then, this device was utilized to monitor changes in $\mathrm{SO}_{2}$ before and after the injection of alcohol and puerarin in mice, starting from $10 \mathrm{~min}$ before ethanol injection. We recorded NIRS signals in the hippocampus for a total duration of $90 \mathrm{~min}$. Oxygen saturation data acquisition lasted for $80 \mathrm{~min}$ post-ethanol administration and $60 \mathrm{~min}$ post-puerarin injection.

\section{T-maze test}

In order to investigate the effects of ethanol consumption with or without puerarin injection on spatial working memory, a T-maze test was performed in mice. The mice were maintained on a restricted diet and kept at $85 \%$ of free-feeding body weight during the behavioral testing in order to maintain the appetitive motivation. The mice first received a 5 -min training session to adapt to the T-maze prior to testing. This training session was carried out 2 times per day for 3 consecutive days by placing 4 grain pellets $(45 \mathrm{mg})$ in the 2 arms of the T-maze. Then, a forced choice training of left-right discrimination was performed 8 times per day for 2 consecutive days. In the study, the arm on the right side of the T-maze was set as the correct one. With the addition of reward pellets in the right arm, the mice were trained to retrieve their spatial working memory and to choose the right direction in the T-maze test. The forced choice training was repeated until the mice achieved a correct choice rate of at least 15/16 for 2 consecutive days. Qualified mice were then administered ethanol and puerarin under various conditions, and the correct choice rate was recorded.

\section{Statistical analysis}

Statistical analysis was performed using SPSS software (SPSS Statistics v. 19.0, IBM Corp., Armonk, USA) by oneway analysis of variance (ANOVA) test and subsequent Dunnett's post-hoc test. Differences were considered to be statistically significant at $\mathrm{p}<0.05$.

\section{Results}

In consideration of the physiological variation of different mice, we normalized the mean firing rate between 0 and 1 in different experiments. As shown in Fig. 2A, the mean firing rate decreased immediately after acute ethanol administration at a dosage of $1.5 \mathrm{~g} / \mathrm{kg}$ (G3), continued declining and reached its lowest point at $20 \mathrm{~min}$ post-injection. It then gradually recovered and returned to its normal level by the 80 min mark. However, with a lower dosage of ethanol of $0.5 \mathrm{~g} / \mathrm{kg}$ (G6), the mean firing rate of neurons remained steady and caused a slight increase at $20 \mathrm{~min}$ post-injection. The effects of puerarin (G2) by itself on the mean firing rate were also studied, 
A


C

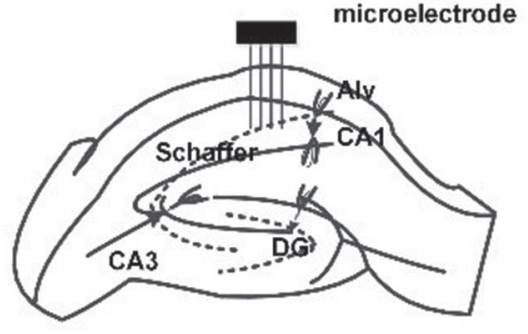

D

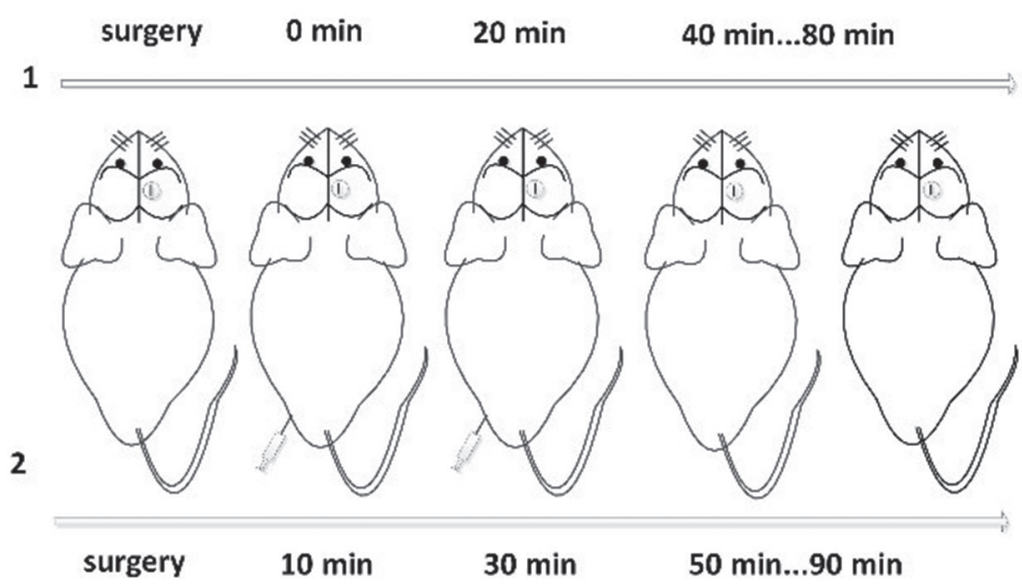

Fig. 1. Experimental setup of electrophysiological signal and $\mathrm{SO}_{2}$ recording

A - schematic diagram of multi-channel data acquisition system; B - schematic illustration of near-infrared spectroscopy (NIRS) monitoring system for oxygen saturation $\left(\mathrm{SO}_{2}\right)$ consisting of: 1 - halogen light source; 2 - fiber optic spectrometer; and 3 - optical fiber probe; $\mathrm{C}$ - the location of the microelectrode in the hippocampus of mice; D - experiment procedure including: 1 - electrophysiological experiment and 2 - NIRS experiment (the dashed circles present the location of the hole in the skull).

and it is suggested that puerarin alone may also alter the mean firing rate with a decreasing tendency.

After ethanol exposure, with the supplementation of puerarin, significant changes in the mean firing rate of neurons in the hippocampal region were found. As shown in Fig. $2 \mathrm{~B}$ and $2 \mathrm{C}$, in the mice injected with ethanol in a higher dosage (G4 and G5), a higher mean firing rate was observed with the involvement of puerarin. In addition, a higher dose of puerarin $(50 \mathrm{mg} / \mathrm{kg})$ caused a higher mean firing rate. However, as to the mice injected with ethanol in a lower dosage (G7 and G8), the mean firing rate was lower with the combination of puerarin. The effects of puerarin are also dose-dependent, with the higher dosage of puerarin $(50 \mathrm{mg} / \mathrm{kg}$ ) causing a significantly lower mean firing rate. 

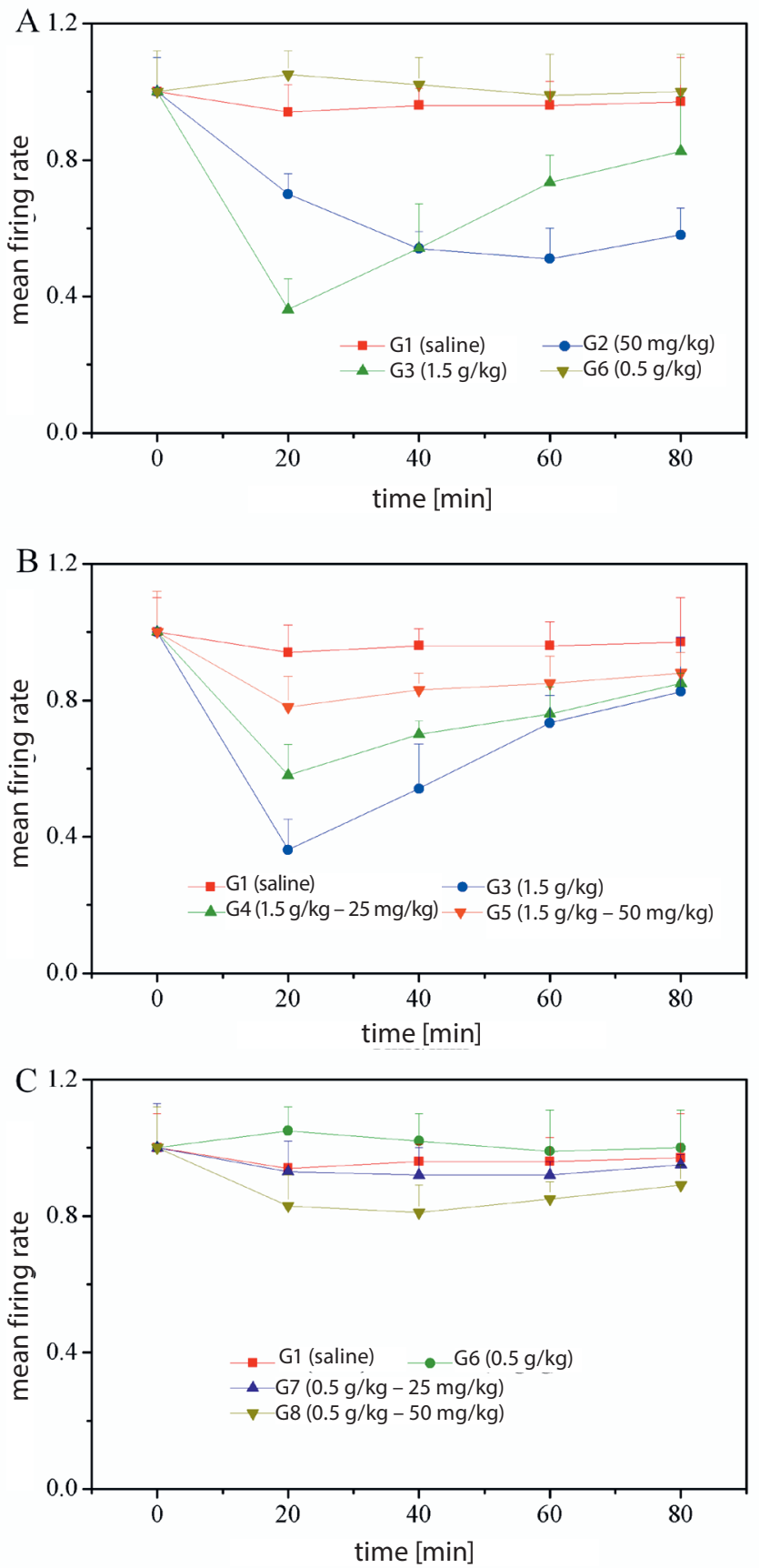

Fig. 2. Mean firing rate

A - mice injected with ethanol in higher $(1.5 \mathrm{~g} / \mathrm{kg})$ and lower $(0.5 \mathrm{~g} / \mathrm{kg})$ dosages; B - mice injected with a combination of ethanol in a higher dosage $(1.5 \mathrm{~g} / \mathrm{kg}$ ) and puerarin (25 and $50 \mathrm{mg} / \mathrm{kg}) ; \mathrm{C}$ - mice injected with a combination of ethanol in a lower dosage $(0.5 \mathrm{~g} / \mathrm{kg})$ and puerarin (25 and $50 \mathrm{mg} / \mathrm{kg}$ ); mice injected with saline $(1.5 \mathrm{~g} / \mathrm{kg}$ ) were used as the control group; the mean firing rate of mice injected only with puerarin $(50 \mathrm{mg} / \mathrm{kg})$ is also presented.

To investigate the effects of puerarin on mice following acute alcohol consumption, $\mathrm{SO}_{2}$ was measured after various treatments. Considering the individual differences, $\mathrm{SO}_{2}$ data was normalized to the $\mathrm{SO}_{2}$ value at the $1^{\text {st }}$ sampling time-point. As shown in Fig. 3A, the normalized $\mathrm{SO}_{2}$ in G3, which was injected with ethanol in a higher dosage $(1.5 \mathrm{~g} / \mathrm{kg})$, was significantly lower $(\mathrm{p}<0.05)$ than that of $\mathrm{G} 1$ (saline), while $\mathrm{SO}_{2}$ showed a significant increase $(\mathrm{p}<0.05)$ in mice from G6, injected with ethanol in a lower dosage
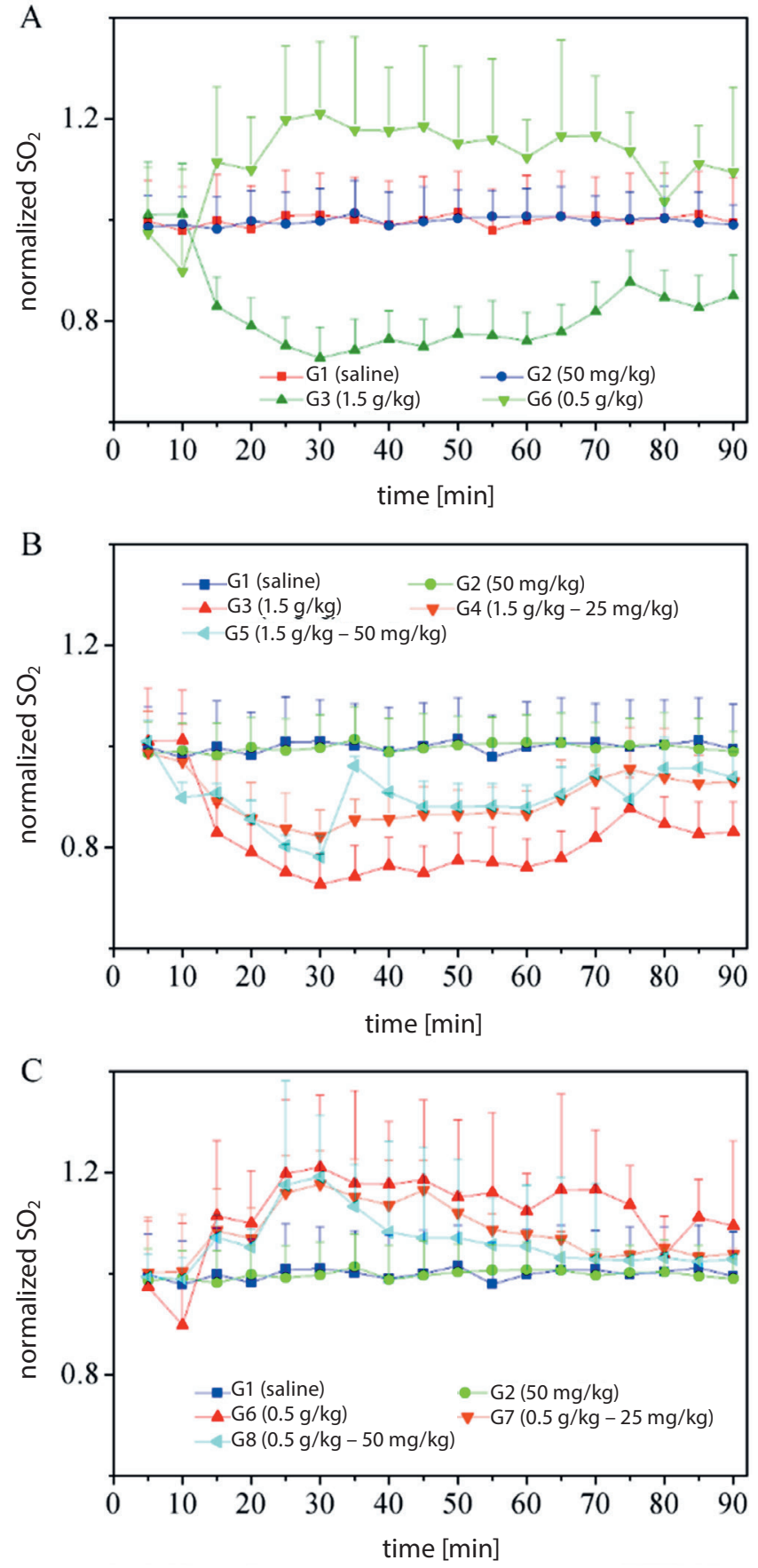

Fig. 3. Normalized oxygen saturation $\left(\mathrm{SO}_{2}\right)$

A - mice injected with ethanol in higher $(1.5 \mathrm{~g} / \mathrm{kg})$ and lower $(0.5 \mathrm{~g} / \mathrm{kg})$ dosages; B - mice injected with a combination of ethanol in a higher dosage $(1.5 \mathrm{~g} / \mathrm{kg}$ ) and puerarin (25 and $50 \mathrm{mg} / \mathrm{kg}) ; \mathrm{C}$ - mice injected with a combination of ethanol in a lower dosage $(0.5 \mathrm{~g} / \mathrm{kg})$ and puerarin (25 and $50 \mathrm{mg} / \mathrm{kg}) ;$ mice injected with saline $(1.5 \mathrm{~g} / \mathrm{kg}$ ) were used as the control group; the normalized $\mathrm{SO}_{2}$ of mice injected only with puerarin $(50 \mathrm{mg} / \mathrm{kg}$ ) is also presented; in each group, data at 10-minute intervals was collected for a baseline, and puerarin at various concentrations was injected 20 min after ethanol administration.

$(0.5 \mathrm{~g} / \mathrm{kg})$. Similar to the negative control group, puerarin by itself showed no obvious changes in $\mathrm{SO}_{2}$ in mice. In Fig. 3B, under ethanol consumption in a higher dosage $(1.5 \mathrm{~g} / \mathrm{kg})$, the $\mathrm{SO}_{2}$ level increased significantly with the supplementation of puerarin in both dosages $(25 \mathrm{mg} / \mathrm{kg}$ 
and $50 \mathrm{mg} / \mathrm{kg}$ ). Meanwhile, $\mathrm{SO}_{2}$ recovered more quickly in the group treated with puerarin in a higher dosage (G5) 10 min after injection.

In Fig. $3 \mathrm{C}$, the $\mathrm{SO}_{2}$ value in $\mathrm{G} 6$ is higher than that of $\mathrm{G} 1$. Then, with the supplementation of puerarin in various dosages (G7 and G8), the $\mathrm{SO}_{2}$ value decreased obviously. The tendency of $\mathrm{SO}_{2}$ in Fig. $3 \mathrm{C}$ is opposite to that presented in Fig. 3B.

To evaluate the effects on the spatial memory of mice after ethanol and puerarin administration, a T-maze test was performed; the results are presented in Fig. 4. The correct choice rate of mice in G1 (saline injection, $1.5 \mathrm{~g} / \mathrm{kg}$ ) did not show an obvious change during the entire time period, while in mice treated with puerarin (G2), the correct choice rate decreased in the first $60 \mathrm{~min}$, and then increased gradually, as shown in Fig. 4A.

As shown in Fig. 4B, following ethanol consumption, a higher dosage of puerarin (G5; $50 \mathrm{mg} / \mathrm{kg}$ ) resulted in a significant increase in the correct choice rate in mice compared to the ethanol group (G3), while the correct choice rate in mice treated with a lower dosage of puerarin (G4; $25 \mathrm{mg} / \mathrm{kg}$ ) was remarkably lower than that of G5. This indicates that, under excessive ethanol consumption, puerarin can resolve the deficit of spatial learning and memory function.

In Fig. $4 \mathrm{C}$, interestingly, the correct choice rate of mice in the ethanol group of the lower dosage (G6; $0.5 \mathrm{~g} / \mathrm{kg}$ ) was consistent with that of the saline group, while the injection of puerarin in ethanol-treated mice led to a decrease in the correct choice rate. This suggests that, in contrary to the above-mentioned results, under excessive alcohol exposure, ethanol in low doses could result in neuronal excitation and an opposite response to puerarin.

\section{Discussion and conclusions}

In our study, electrophysiological signals and $\mathrm{SO}_{2}$ in the hippocampal region of mice were first obtained in vivo before and after the administration of ethanol. As in the case of our previous results, an ethanol dosage of $1.5 \mathrm{~g} / \mathrm{kg}$ inhibited the firing rate of neurons. By inhibiting the function of glutamate receptors and/or altering the balance between inhibitory and excitatory neurotransmission, acute alcohol consumption can cause cognitive impairment in certain brain areas, such as the hippocampus, amygdale and striatum. ${ }^{18}$ Interestingly, however, ethanol at a lower dosage $(0.5 \mathrm{~g} / \mathrm{kg})$ resulted in the opposite tendency. This suggests that the inhibitory effects of ethanol on the brain are highly dependent on the dose of ethanol applied.

In addition to electrophysiological changes, a lot of evidence suggested that alcohol could decrease the affinity of hemoglobin to oxygen, resulting in lower $\mathrm{SO}_{2}$ levels. Alcohol ingestion was reported to increase the incidence of arterial oxygen desaturation and disordered breathing
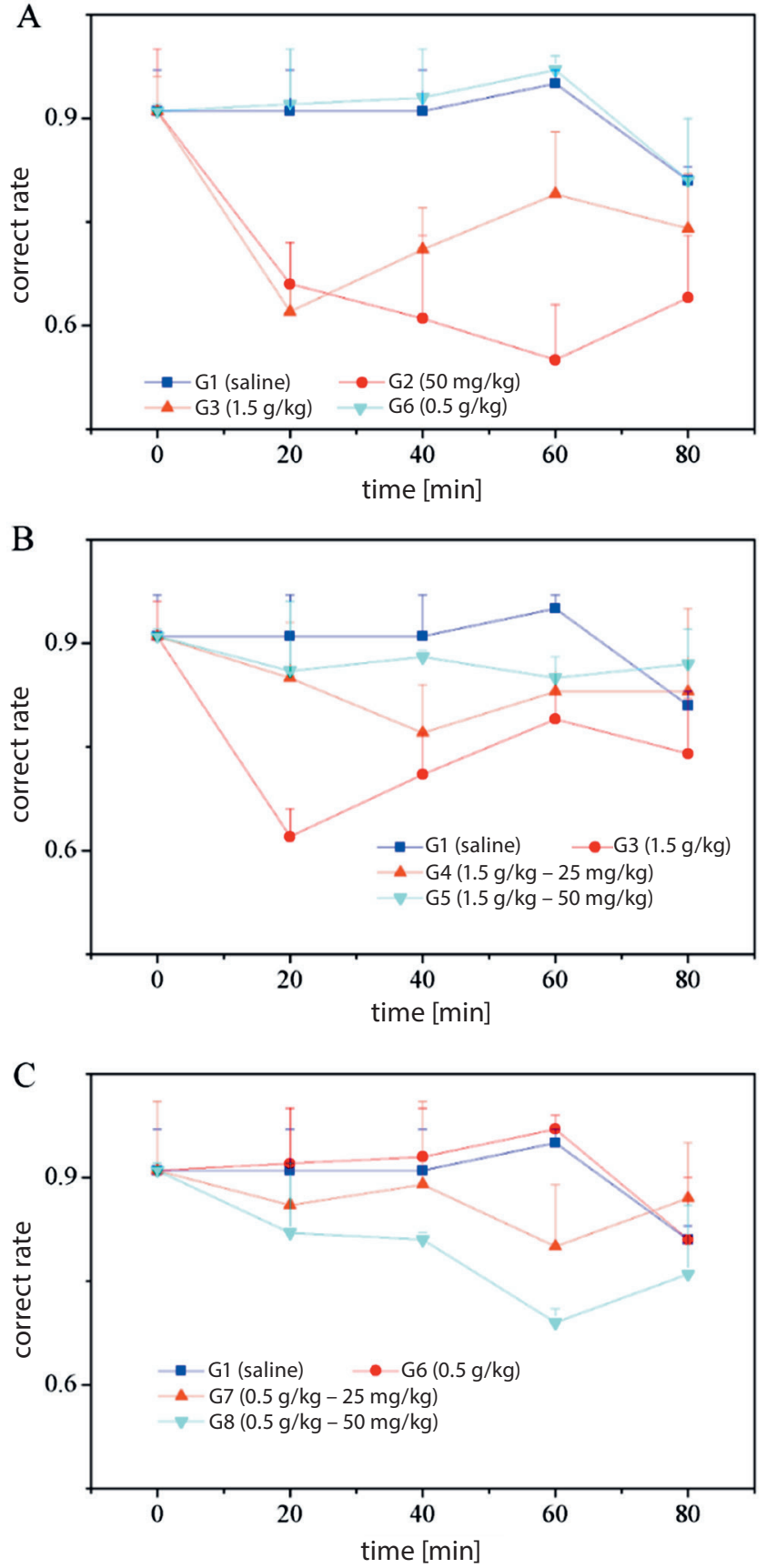

Fig. 4. The average correct choice rate of mice in a T-maze test

A - after ethanol injection alone; B - after ethanol injection in a higher dosage $(1.5 \mathrm{~g} / \mathrm{kg}$ ), followed by puerarin injection (25 and $50 \mathrm{mg} / \mathrm{kg}$ ); $\mathrm{C}$ - after ethanol injection in a lower dosage $(0.5 \mathrm{~g} / \mathrm{kg})$, followed by puerarin injection (25 and $50 \mathrm{mg} / \mathrm{kg}$ ); mice injected with saline $(1.5 \mathrm{~g} / \mathrm{kg}$ ) were used as the control group; the average correct choice rate of mice injected only with puerarin $(50 \mathrm{mg} / \mathrm{kg})$ is also presented.

during sleep. ${ }^{19}$ Therefore, $\mathrm{SO}_{2}$ could be another indicator which reveals the effects of ethanol on the brain and could be used to evaluate the effectiveness of medications in treating alcoholism. By integrating NIRS with an electrophysiological signal recording system, the $\mathrm{SO}_{2}$ values in the hippocampus of mice under various treatments were obtained simultaneously. We found that changes of $\mathrm{SO}_{2}$ were positively correlated with the firing rate. The decrease 
in $\mathrm{SO}_{2}$ was accompanied by a decline in the firing rate of mice injected with ethanol in higher dosages. Interestingly, similar dose-dependence was also found in $\mathrm{SO}_{2}$ results, with higher $\mathrm{SO}_{2}$ appearing in the lower-dosage ethanol group. Indeed, previous experiments have found that ethanol in a moderate dosage of $0.2 \mathrm{~g} / \mathrm{kg}$ and $0.5 \mathrm{~g} / \mathrm{kg}$ could somehow increase the activity of superoxide dismutase (SOD) and decrease the content of malondialdehyde (MDA), thus resulting in an increase of the neuron firing rate and $\mathrm{SO}_{2}$ level. ${ }^{20}$ In contrast, an excessive intake of ethanol caused a significantly greater reduction in $\mathrm{SO}_{2}$ together with an obvious decrease in the firing rate.

In order to assess the effects of ethanol on spatial learning and memory function, we performed a T-maze test on mice under various treatments. Consistent with previous reports, we indeed found that the spatial memory function of mice could be impaired by excessive ethanol injection. Similar decreasing trends in both behavior performance and spike recording were observed in mice exposed to ethanol in a higher dosage $(1.5 \mathrm{~g} / \mathrm{kg})$. The most significant spatial memory dysfunction appeared at the time-point when firing rates changed most drastically. In addition, the present study also provides direct evidence for the association between the impairment of spatial memory function and the changes of electrophysiological signals and $\mathrm{SO}_{2}$. The results of the T-maze test show the same tendency regarding the firing rate and $\mathrm{SO}_{2}$. These findings are consistent with previous results, suggesting that behavioral disorders and hippocampal dysfunctions would appear upon excessive intake of ethanol, while a moderate dosage of ethanol would not impair spatial memory in mice. ${ }^{21}$

Based on many years of clinical experience, Puerariae radix as a Chinese herbal medicine has been widely used to cure alcoholism. ${ }^{22,23}$ Puerarin extracted from Puerariae radix is one of the main effective components which can suppress the severity of alcohol withdrawal symptoms. Previous studies have suggested that puerarin could reduce the ethanol concentration in the blood of Sprague Dawley ${ }^{\circledR}$ (SD) rats with acute ethanol intoxication. ${ }^{24}$ The pharmacological function of puerarin also includes its vasodilative effects, which thereby increase oxygen supply to brain tissues. In our study, the protective effects of puerarin in ethanol-induced brain impairment were investigated by monitoring the typical changes of electrophysiological and $\mathrm{SO}_{2}$ levels, and analyzing behavior performance during treatment. Puerarin had a significant antagonistic effect on the decreased firing rate, $\mathrm{SO}_{2}$ level and correct choice rate upon acute high-dosage ethanol consumption. Previous studies have found that puerarin has strong antioxidant effects. ${ }^{25,26}$ Indeed, in the present study, we found that oxygen desaturation induced by excessive ethanol consumption could be alleviated with the addition of puerarin. It indicated that ethanol-induced brain impairment could be suppressed by treatment with puerarin, as evidenced by the increased firing rate and $\mathrm{SO}_{2}$ level, and improved behavior performance. Besides, the dose-dependent effects of purarin on the firing rate and $\mathrm{SO}_{2}$ are also consistent with previous in vitro findings, showing that cell viability was recovered dose-dependently with puerarin in ethanoltreated cells.

In conclusion, electrophysiological signal analysis and NIRS were combined for the first time to prove that the impairment of spatial learning and memory of mice induced by ethanol is dose-dependent. An excessive consumption of ethanol is required to cause detrimental effects on the spatial memory of mice by modulating electrophysiological transmission and $\mathrm{SO}_{2}$ in brain tissue. Our research showed that puerarin could inhibit ethanol-induced brain impairment by ameliorating oxidative reactions in vivo against the adverse effect of ethanol injection. These findings highlight the significance of our study in providing an innovative approach to evaluate the use of the medication in the treatment of alcohol-induced brain disorders.

\section{References}

1. Constant A, Sherlaw W, Kovess-Masfety V. Seeking mental health care from private health practitioners among individuals with alcohol dependence/abuse results from a study in the French general population. Alcohol. 2017;59:1-6.

2. Wimmer, GE, Shohamy D. Preference by association: How memory mechanisms in the hippocampus bias decisions. Science. 2012; 338(6104):270-273.

3. Elibol-Can B, Kilic E, Yuruker S, Jakubowska-Dogru E. Investigation into the effects of prenatal alcohol exposure on postnatal spine development and expression of synaptophysin and PSD95 in rat hippocampus. Int J Dev Neurosci. 2014;33:106-114.

4. Zou, Y, Hong B, Fan L, Zhou L, Liu Y, Wu Q. Protective effect of puerarin against beta-amyloid-induced oxidative stress in neuronal cultures from rat hippocampus: Involvement of the gsk-3 $\beta / \mathrm{nrf} 2$ signaling pathway. Free Radic Res. 2013;47(1):55-63.

5. Zhang R, Hu Y, Yuan J, Wu D. Effects of puerariae radix extract on the increasing intestinal permeability in rat with alcohol-induced liver injury. J Ethnopharmacol. 2009;126(2):207-214.

6. Liu CM, Ma JQ, Sun YZ. Puerarin protects rat kidney from lead-induced apoptosis by modulating the pi3k/akt/enos pathway. Toxicol Appl Pharmacol. 2012;258(3):330-342.

7. Zhao SS, Yang WN, Jin H, Ma KG, Feng GF. Puerarin attenuates learning and memory impairments and inhibits oxidative stress in STZ-induced SAD mice. Neurotoxicology. 2015;51:166-171.

8. Lee $\mathrm{O}$. Effects of supplementation of puerariae radix ethanol extract on the antioxidative defense system in rats. Korean J Nutr. 2004;37: 872-880.

9. Wu HQ, Guo HN, Wang HQ, Chang MZ, Zhang GL, Zhao YX. Protective effects and mechanism of puerarin on learning-memory disorder after global cerebral ischemia-reperfusion injury in rats. Chin J Integr Med. 2009;15(1):54-59.

10. Jonsson S, Ericson M, Söderpalm B. Modest long-term ethanol consumption affects expression of neurotransmitter receptor genes in the rat nucleus accumbens. Alcohol Clin Exp Res. 2014;38(3):722-729.

11. Shahidi S, Zargooshnia S, Asl SS, Komaki A, Sarihi A. Influence of $\mathrm{N}$-acetyl cysteine on beta-amyloid-induced Alzheimer's disease in a rat model: A behavioral and electrophysiological study. Brain Res Bull. 2017;131:142-149.

12. Horvath G, Petrovszki Z, Kekesi G, et al. Electrophysiological alterations in a complex rat model of schizophrenia. Behav Brain Res. 2016;307:65-72.

13. Chernov MM, Chen G, Torre-Healy LA, Friedman RM, Roe AW. Microelectrode array stimulation combined with intrinsic optical imaging: A novel tool for functional brain mapping. J Neurosci Meth. 2016;263:7-14 
14. Migliorini R, Moore EM, Glass L, et al. Anterior cingulate cortex surface area relates to behavioral inhibition in adolescents with and without heavy prenatal alcohol exposure. Behav Brain Res. 2015;292:26-35.

15. Qian Z, Victor SS, Gu Y, Giller CA, Liu H. "Look-ahead distance" of a fiber probe used to assist neurosurgery: Phantom and Monte Carlo study. Opt Express. 2003;11:1844-1855.

16. Li W, Qian Z, Li T. Method to improve the performance of reflectance diffuse optical imaging based on polygonal optical fibers arrangement. Chin Opt Lett. 2009;7:852-856.

17. Yubing L, Hongke W, Yangyang L, Weitao L, Zhiyu Q. Monte Carlo and phantom study in the brain edema models. J Innov Opt Health Sci. 2017;10(3):1-11.

18. Matthews DB, Simson PE, Best PJ. Ethanol alters spatial processing of hippocampal place cells: A mechanism for impaired navigation when intoxicated. Alcohol Clin Exp Res. 1996;20(2):404-407.

19. Carole W, Sherry L, Taasan VC, Block AJ, Boysen PG, Wynne JW. Alcohol increases sleep apnea and oxygen desaturation in asymptomatic men. Am J Med. 1981;71(2):240-245.

20. Zhao SM, Huang JJ, Huang LJ, Xian-Jiao HE, Jin L. Effects of different dose alcohol on the sod activity and mda content in rabbits. Journal of Youjiang Medical College for Nationalities. 2000;22(05):689-690.
21. Novier A, Van Skike CE, Chin VS, Diazgranados JL, Matthews DB. Low and moderate doses of acute ethanol do not impair spatial cognition but facilitate accelerating rotarod performance in adolescent and adult rats. Neurosci Lett. 2012;512(1):38-42.

22. Liu Y, Yang Y, Sun M. Highly specific noninvasive photoacoustic and positron emission tomography of brain plaque with functionalized croconium dye labeled by a radiotracer. Chem Sci. 2017;8(4):2710-2716.

23. Nie L, Guo Z, Wang LV. Photoacoustic tomography of monkey brain using virtual point ultrasonic transducers. J Biomed Opt. 2011;16(7): 076005.

24. Barry DI, Hemmingsen R. Cerebral blood flow autoregulation during acute ethanol intoxication in the rat. Acta Pharmacol Toxicol (Copenh). 1984;54(3):227-232.

25. Ronis MJ, Hennings L, Stewart B, Basnakian AG, ApostolovEO, AlbanoE. Effects of long-term ethanol administration in a rat total enteral nutrition model of alcoholic liver disease. Am J Physiol Gastrointest Liver Physiol. 2011;300(1):109-119.

26. Noh BK. Restoration of autophagy by puerarin in ethanol-treated hepatocytes via the activation of AMP-activated protein kinase. Biochem Biophys Res Commun. 2011;414(2):361-366. 